\title{
Utilizando simulação computacional como estratégia de ensino: estudo de caso
}

\author{
João Artur de Souza - UFSC - jartur@egc.ufsc.br \\ Gertrudes Aparecida Dandolini - UFSC - gtude@egc.ufsc.br
}

Resumo: Este artigo descreve uma estratégia de ensino aprendizagem utilizando simulação computacional. A proposta é a utilização de software de matemática (como SCILAB ou MATLAB®) para realizar simulações de problemas de Engenharia. Neste artigo, os alunos optaram por estudar o problema da qualidade na produção de cerâmica vermelha. Para isso, foram orientados a estudarem a teoria dos conjuntos difusos, sistemas especialistas e lógica de programação a fim de desenvolverem simulações através do software. Os primeiros resultados evidenciam que a estratégia pode de fato proporcionar uma aprendizagem significativa dos conceitos envolvidos no processo.

Palavras-chave: aprendizagem significativa, simulação

\section{Using computer simulation as a education strategy: a case study}

Abstract: This article describes a teaching strategy for learning using computer simulation. The proposal is the use of mathematical software (such as MATLAB or Scilab $\left.{ }^{\circledR}\right)$ to perform simulations of engineering problems. In this article, the students chose to study the problem of quality in the production of red ceramic. For that, were instructed to study fuzzy sets, expert systems and logic programming in order to develop simulations using the software. The first results show that the strategy may actually provide a meaningful learning of concepts involved in the process.

Keywords: meaningful learning, simulation

\section{Introdução}

Segundo MORAN (2004) "Uma das reclamações generalizadas de escolas e universidades é de que os alunos não agüentam mais nossa forma de dar aula. Os alunos reclamam do tédio de ficar ouvindo um professor falando por horas,...”. Isso significa que algo está errado e precisa ser mudado. E as mudanças necessárias conduzem a um repensar nos papéis dos envolvidos no processo ensino-aprendizagem e também nos métodos utilizados.

A sociedade vive momentos de grandes transformações. Passamos da sociedade industrial para a sociedade da informação (ou do conhecimento) onde as fontes fundamentais de riqueza são o conhecimento e a comunicação (Stewart, 1998). Desta forma, precisamos ministrar nossas aulas, elaborar nossos currículos para preparar nossos alunos para essa nova sociedade. $\mathrm{O}$ mercado de trabalho busca por profissionais críticos e criativos, que possuam conhecimentos e que tenham consciência deste. $\mathrm{Ou}$ seja, ele deve ter clareza sobre o conhecimento que domina. Saber o que sabe.

Nesta direção, a introdução de modelos de ensino/aprendizagem flexíveis, adaptáveis ao perfil e estilo de aprendizagem dos alunos vem contribuindo enormemente para uma aprendizagem significativa (Moreira, 1999), no sentido de prover argumentos que 
evidencie ao aluno o papel importante do domínio do conteúdo (conhecimento) e o saber fazer na sua formação, por exemplo. A utilização de idéias novas que possam se transformar em modelos pedagógicos é hoje um desafio para as Instituições de Ensino Superior.

Tem se tornado evidente que as Instituições de Ensino, nas suas políticas e iniciativas de modernização do processo de ensino, devem adotar estratégias que apontem para o ensino a distância ou semipresencial, que permita aos alunos manterem um alto grau de envolvimento com o seu curso sem necessidade de se deslocarem frequentemente às instituições. Neste nosso trabalho, ainda não estaremos nos reportando a este fato. Estamos numa primeira etapa, que é o envolvimento presencial. A importância desta primeira fase se justifica pelo alto índice de desinteresse dos alunos aos seus cursos (Censo da Educação Superior 2007 divulgado pelo Ministério da Educação - MEC).

Uma estratégia adotada aqui na UFSC no curso de Engenharia de Materiais é a realização de vários estágios durante o desenvolvimento do curso. $\mathrm{O}$ curso é executado em 15 trimestre (5 anos) e a partir do $5^{\circ}$ trimestre iniciam os estágios. Os estágios são intercalados com trimestres de disciplinas. Assim, a partir do segundo ano de curso, o aluno fica um semestre na universidade cursando disciplinas e um trimestre na empresa. $\mathrm{O}$ que se observa com esta estratégia, são alunos muito mais preocupados em experimentar as mais variadas situações durante as disciplinas. Os estágios dão diretrizes ao aluno e os motivam na busca da construção de um saber prático. Isso leva o professor a pesquisar sobre como proceder para mostrar a importância do conhecimento teórico que dá base para o saber fazer.

Nesta direção, estamos trabalhando na elaboração de aulas que levem os alunos ao desenvolvimento de situações que resultem na construção dos conceitos. O maior indicador da preocupação do curso é o reconhecimento da importância no aumento da qualidade do processo ensino/aprendizagem e no combate ao desinteresse acadêmico.

Para alcançar o objetivo dividiremos este artigo em alguns tópicos: aprendizagem significativa e simulação, modelo e aplicação do modelo e resultados.

\section{Aprendizagem Significativa e Simulação}

A teoria sobre aprendizagem significativa foi desenvolvida pelo psicólogo norteamericana David Ausubel. Segundo ele a essência do processo de aprendizagem significativa é que idéias simbolicamente expressas sejam relacionadas de maneira substantiva (não literal) e não arbitrária ao que o aprendiz já sabe, ou seja, a algum aspecto de sua estrutura cognitiva especificamente relevante para a aprendizagem dessas idéias.

A aprendizagem significativa ocorre quando o aluno consegue mesclar o conhecimento prévio ao conhecimento novo de forma que haja uma evolução no seu conhecimento. Moreira coloca que na aprendizagem significativa:

"há uma interação entre o novo conhecimento e o já existente, na qual ambos se modificam. À medida que o conhecimento prévio serve de base para a atribuição de significados à nova informação, ele também se modifica, ou seja, os subsunçores vão adquirindo novos significados, se tornando mais diferenciados, mais estáveis. Novos subsunçores vão se formando; subsunçores vão interagindo entre si. A estrutura cognitiva 
está constantemente se reestruturando durante a aprendizagem significativa. $O$ processo é dinâmico; o conhecimento vai sendo construído. (Moreira, 1998)

Segundo Pellizari et al (2002) é necessário para que a aprendizagem significativa ocorra:

Em primeiro lugar o aluno precisa ter uma disposição para aprender: se o indivíduo quiser memorizar o conteúdo arbitrária e literalmente, então a aprendizagem será mecânica. Em segundo, o conteúdo escolar a ser aprendido tem que ser potencialmente significativo, ou seja, ele tem que ser lógica e psicologicamente significativo: o significado lógico depende somente da natureza do conteúdo, e o significado psicológico é uma experiência que cada indivíduo tem. Cada aprendiz faz uma filtragem dos conteúdos que têm significado ou não para si próprio.

Neste sentido é que buscamos, através de simulações computacionais de problemas definidos pelos próprios alunos, criar as condições necessárias para que a aprendizagem significativa ocorra.

As atividades de modelagem e simulação estão entre as formas mais criativas, benéficas e que geram interação de aplicações na Educação. A razão principal é que a construção e simulação de modelos têm muito a ver com o modo como o próprio conhecimento científico é testado e desenvolvido, através do ciclo: hipóteses, observação e planejamento, experimentação, e formação ou comprovações de leis e teorias (ADAO e Bernardino, 2003). Além disso, os sistemas computacionais fornecem meios de cálculo e representação que tornam possível o uso didático da simulação, sem necessidade de habilidades especiais em programação matemática. O computador agrega um elemento de realismo, que motiva de forma dinâmica o aluno, principalmente quando consegue desenvolver as simulações.

Existem muitas definições para simulação. Utilizamos aqui a definição de Pegden (1991) onde "simulação é processo de projetar um modelo computacional de um sistema real e conduzir experimentos com esse modelo com o propósito de entender seu comportamento e/ou avaliar estratégia para a sua operação."

\section{Modelo}

Os cursos de Engenharias devem preparar o aluno para o mercado de trabalho. Observase aqui a necessidade do aluno sair com experiências adversas para enfrentar os desafios que a realidade irá impor. Desta forma, devemos prepará-los para enfrentar esses desafios, tornando-os capazes de gerenciar e aplicar os seus conhecimentos adquiridos durante o curso. Assim, o estudo de situações práticas através de simulações é uma estratégia importante e vital. Lembrando que a simulação apresenta-se como uma forma de aprimorar a aprendizagem dos conceitos, de desenvolver novos conhecimentos e também como um meio de motivar os alunos.

A simulação como estratégia foi, a princípio, uma forma encontrada de fazer com que os alunos se envolvessem e se comprometessem mais com as disciplinas. Definimos então um modelo de como ministraríamos a disciplina. Num primeiro momento seria lançado o desafio aos alunos de buscarem problemas de interesse e que envolvesse de forma direta a formação deles. A partir desses problemas seriam escolhidos aqueles que pudessem ser tratados com as técnicas que a disciplina se propunha a estudar. Definidos 
os problemas, o professor orienta os alunos nos conteúdos que ele precisa conhecer para resolver o problema e após ser capaz de modelar e fazer a simulação.

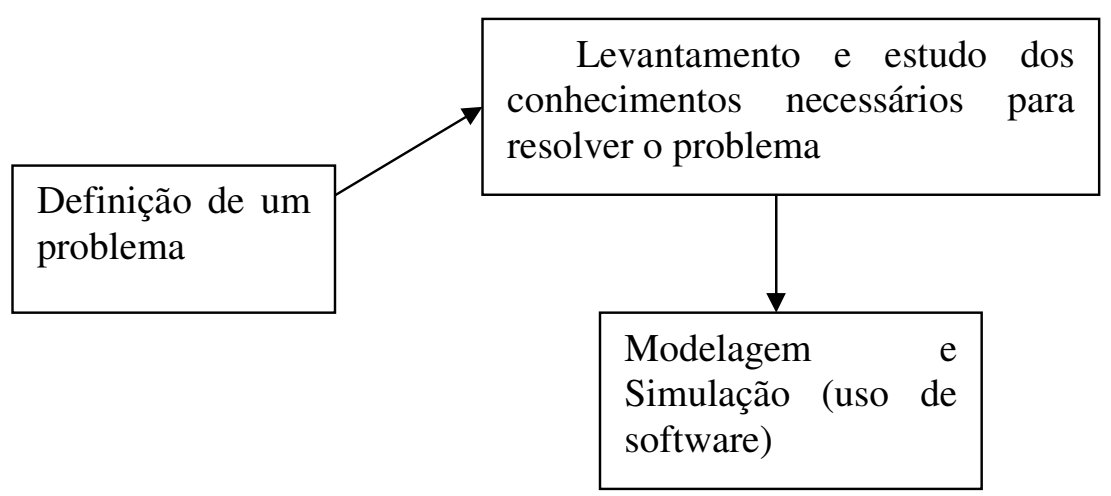

Figura 1: Modelo da estratégia utilizada

O modelo (Figura 1) se converte em estratégia a partir de uma divisão de competências aplicadas. O modelo é definido como um sistema de aprendizagem interativo suportado pelo ambiente apresentado pelo software de matemática que envolve uma estrutura integrada de recursos de apoio de simulação e o apoio didático pedagógico enfatizado nas estratégias conceituais. A modelagem e simulação podem ser usadas em uma grande variedade de contextos no processo ensino-aprendizagem.

Considerando a definição anterior nos referimos ao modelo através de três vetores essenciais:

Aprendizagem Interativa - Evidencia a forte interação do aluno com conteúdo abordado e com o professor. Esta é uma característica fundamental que enfatiza o interesse despertado com a orientação adequada para o alcance do proposto.

Ambiente de Simulação - O suporte através de tecnologia que integra um conjunto de serviços e ferramental para criação de condições para a realização de atividades pedagógicas. Provocando a familiarização do aluno com ferramentas que podem ser de utilidade ilimitada no decorrer do curso. Estes softwares, assim como muitos outros que existem, integra num único ambiente a simplicidade da ferramenta e a utilidade estratégica no interesse e entendimento conceitual.

Estratégia Pedagógica - Significa, basicamente, que todos os conteúdos e atividades pedagógicas são especificamente desenhados para o auto-estudo, a motivação, autoaprendizagem sob orientação estratégica, fornecendo mecanismos de avaliação de progresso, de consolidação de conhecimentos e de auto-avaliação.

\section{Aplicação do modelo e resultados}

Aplicamos a estratégia da simulação na disciplina de Tecnologias Aplicadas a Engenharia que tem como objetivo proporcionar aos alunos conhecimentos para conceber, modelar, analisar e controlar processos e atividades, não apenas quanto ao essencial das potencialidades tecnológicas, mas também quanto às suas implicações organizacionais, tácticas e estratégicas. Após concluir esta disciplina, o aluno deve dispor de conhecimentos de referência para aprimorar o sistema de informação, integrando conceitos com modelagem apropriada. A principal ferramenta utilizada na disciplina são softwares de matemática que usam linguagem interpretada para simular os objetivos propostos. Os softwares utilizados são basicamente o SCILAB e o MATLAB®. 
Para atingir os objetivos propostos, foram desenvolvidas estratégias que mostrassem as diferentes fases do processo. Com o objetivo de manter no aluno um alto grau de envolvimento no processo de ensino aprendizagem, as atividades tiveram caráter flexível, interativo e participativo, garantindo sempre que os objetivos da disciplina fossem alcançados.

Os conteúdos foram desenhados para promover a pesquisa, tendo como referência os objetivos: avaliar a aceitação e o grau de satisfação dos alunos com esse novo método; inventariar outros recursos necessários para o desenvolvimento sistemático de ações; definir estratégias mais adequadas; aumentar o interesse do aluno no seu curso; valorizar mais o seu curso para a sua formação e estimular uma cultura de responsabilidade pelo processo de aprendizagem.

Uma preocupação com respeito ao aluno refere-se a sua capacidade de auto-estudos para preparar novas simulações. Entretanto, esta proposição não foi o objeto de estudo nesta primeira etapa, ficando como um tópico para estudos futuros. Para contornar esse possível problema foi utilizado a estratégia de estudo colaborativo. Como apoio ás dúvidas eventuais foi utilizado um ambiente virtual de aprendizagem (Moodle ${ }^{1}$ ) como apoio não presencial.

O desenvolvimento do conteúdo seguiu as instruções das atividades. Foi definido um problema inicial, no caso, a qualidade na produção de cerâmica vermelha. Em seguida, após estudo do problema, foram planejadas as atividades intermediárias, que consistiam no estudo da teoria de conjuntos difusos, sistemas de regras, e lógica de programação. A proposta da simulação como fonte motivadora e de aprendizagem, levou o aluno ao centro no processo da aprendizagem. Iniciou então o estudo do software para em seguida desenvolver as simulações. Essa fase foi mais complexa, pois o conhecimento dos alunos não era homogêneo em relação a programação no software. Para contornar esse problema, foram elaboradas algumas atividades que consistiam na resolução de problemas mais conhecidos com auxílio do software. Desta forma, os alunos se sentiam motivados a resolver os problemas e com isso aprendiam a manusear o software, mostramos os recursos potenciais do software.

O problema exemplo desenvolvido foi o descrito no artigo de Neto e Castanho (2008) onde os autores discutem a utilização de conjuntos difusos para modelar a avaliação da qualidade na produção da cerâmica vermelha. Os alunos fizeram então a simulação no toolboxFuzzy do Matlab® e puderam experimentar o significado de cada característica discutida no artigo. Os autores discutem os fatores que influenciam para a qualidade da cerâmica vermelha, tais como: Matéria prima, condições ambientais, tempo de secagem e queima. No artigo eles comentam e referenciam os estudos que mostram a influência dos fatores, assim, foram definidas regras na toolboxfuzzy do Mtlab® para proceder as simulações. O exemplo tinha por objetivo, não só mostrar a necessidade do aprendizado, neste caso, da toolbox de difuso e entendimento da teoria de conjuntos difusos, como também a influência dos fatores na qualidade da cerâmica produzida. A Figura 2 abaixo mostra a tela do Matlab ${ }^{\circledR}$ com a simulação desenvolvida por um dos grupos dos alunos.

\footnotetext{
${ }^{1} \mathrm{O}$ Moodle é um ambiente virtual de aprendizagem livre e mundialmente utilizado. Para maiores informações visite a pagina http://moodle.org/
} 


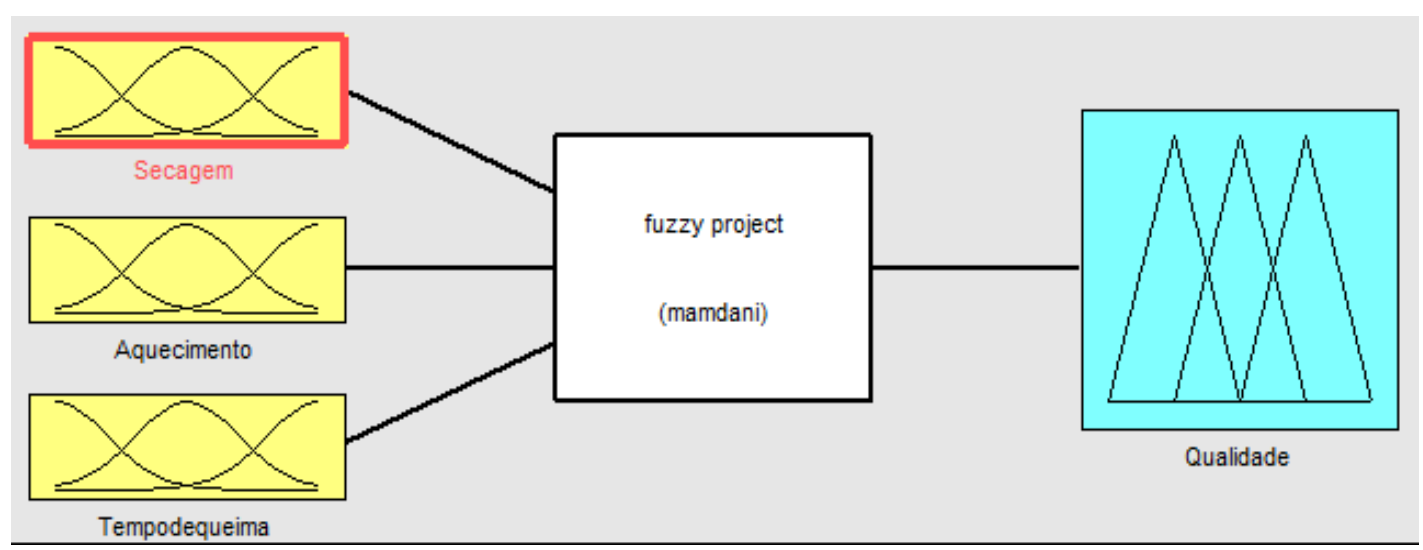

Figura 2: Modelo desenvolvido no toolbox do Matlab® por um dos grupos dos alunos

Para apresentar este modelo, algumas regras tiveram de ser programadas. A Figura 3 abaixo mostra algumas regras programadas.

Rule Editor: fuzzy project

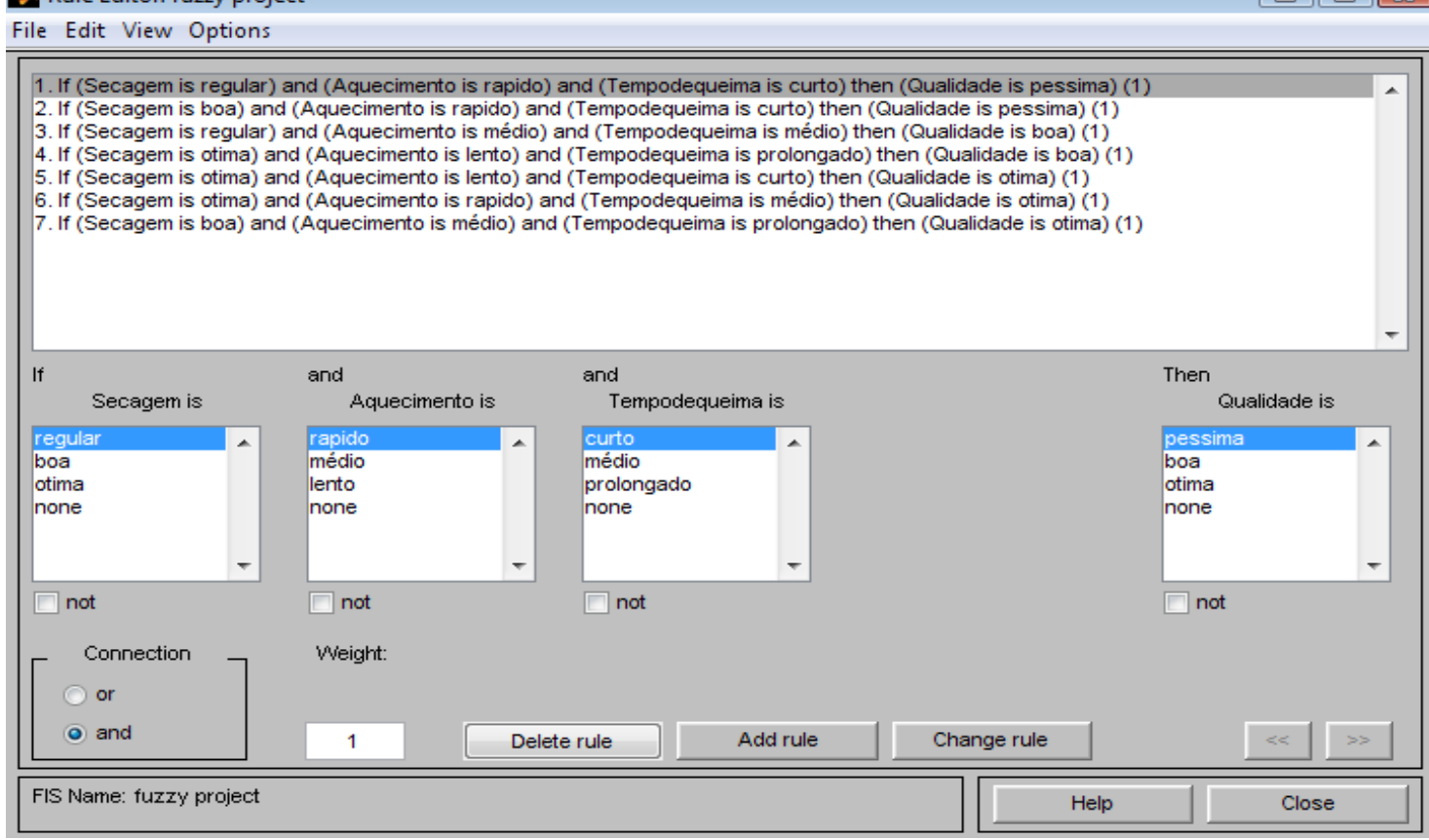

Figura 3: Tela apresentada pelo matlab® ${ }^{\circledR}$ com as regras

Algumas das regras implementadas são mostradas na Tabela 1.

Tabela 1: Apresentação da relação entre os fatores desenvolvidos baseados no artigo de Neto e Castanho (2008)

\begin{tabular}{|l|l|l|l|}
\hline Secagem & Taxa de Aquecimento & Tempo de Queima & Qualidade \\
\hline Regular & Rápido & Curto & Péssima \\
\hline Boa & Rápido & Curto & Péssima \\
\hline Regular & Médio & Médio & Boa \\
\hline Ótima & Lento & Prolongado & Boa \\
\hline
\end{tabular}




\begin{tabular}{|l|l|l|l|}
\hline Ótima & Lento & Curto & Ótima \\
\hline Ótima & Rápido & Médio & Ótima \\
\hline Boa & Médio & Prolongado & Ótima \\
\hline
\end{tabular}

Observe que este exemplo provocou o estudo não só de conjuntos fuzzy, como também, sistemas especialistas, formação de regras, assim como o entendimento de lógica. $\mathrm{O}$ uso do software Matlab® também ofereceu durante o seu estudo, muito entusiasmo aos alunos devido sua facilidade de aprendizado. Além disto, o aluno envolveu nesta disciplina um conhecimento de outra disciplina do seu curso e que são relevantes na sua formação, no caso qualidade de cerâmica. Para fazer as simulações ele teve que explicitar, neste caso, através das regras, o conhecimento adquirido em outro momento de seu curso.

Durante o desenvolvimento das simulações ficou claro o entendimento das teorias adjacentes, no caso, conjuntos difusos. Observe a construção das funções de pertinência e escala de valores. Nas figuras abaixo, pode-se perceber o desenvolvimento realizado.

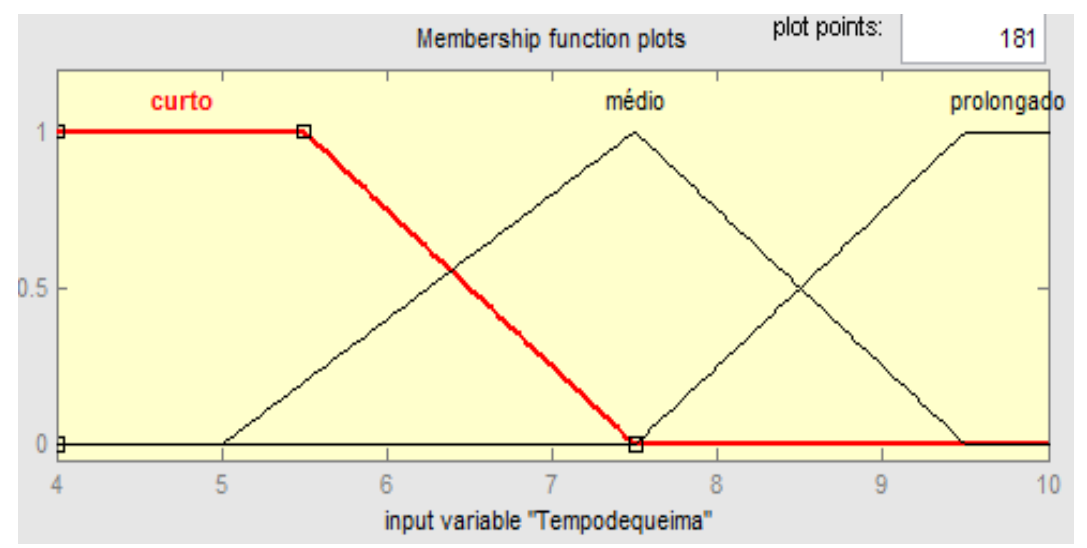

Figura 4: Funções de pertinências para a variável tempo de queima: o tempo foi dividido em três estágios, segundo as orientações do problema, ficando: tempo curto; tempo médio e tempo prolongado. Para cada um foi definido a função de pertinência, trapezoidal, triangular e trapezoidal, respectivamente

A qualidade da cerâmica foi modelada como "péssima", "boa" e "ótima" e com as funções de pertinências triangulares como pode ser observadas na Figura 5. 


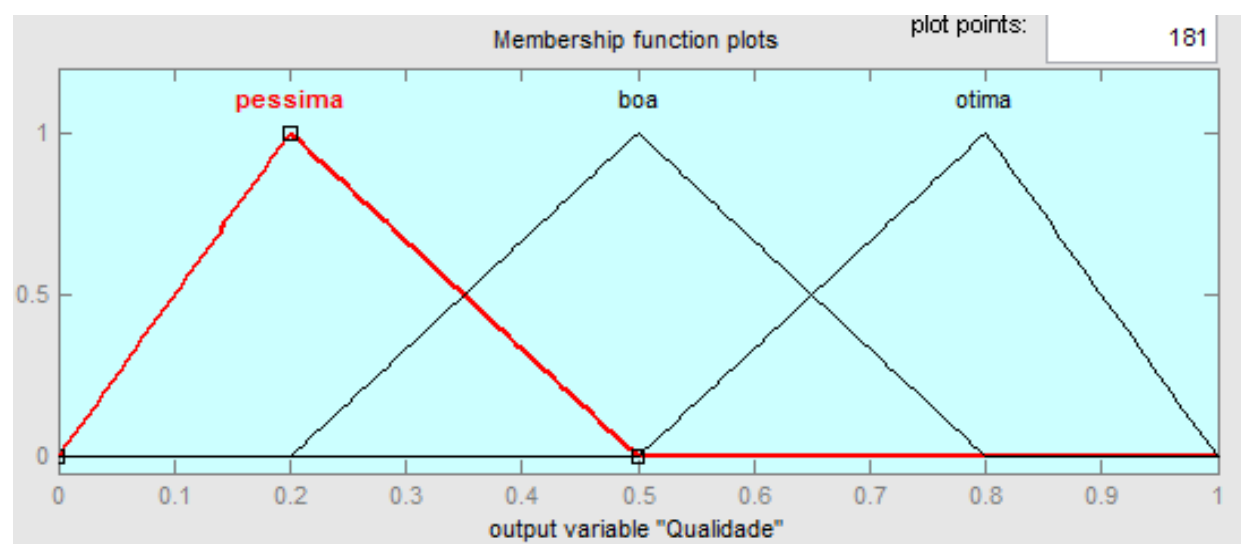

Figura 5: Funções de pertinências para a variável qualidade

Os alunos tiveram a oportunidade de experimentar e discutir como cada regra influenciava na qualidade final da cerâmica vermelha. $\mathrm{O}$ nível de envolvimento foi intenso, o que leva a avaliar esta estratégia como promissora neste tipo de estudo.

Nesta disciplina, foram simulados outros problemas para abranger outros conteúdos da disciplina.

Os resultados da aplicação do modelo, neste primeiro momento, superaram as expectativas. De fato houve um grande envolvimento por parte dos alunos na disciplina que, para alcançarem o objetivo de resolver e simular o problema proposto, estudaram e aprenderam mais conceitos que o previsto pela disciplina.

\section{Considerações finais}

O projeto de usar a simulação como estratégia de ensino aprendizagem surtiu efeito desde o seu início, criando expectativas e interesses nos alunos não observados antes. Além disto, o resultado da aprendizagem mostrou-se evidente no momento em que os alunos conseguiram simular a resolução do problema proposto e discutiam os resultados obtidos. Porém, essa estratégia foi utilizada em apenas uma turma, e a análise dos resultados foram realizadas sem os rigores de uma pesquisa científica.

Como trabalho futuro, pretendemos realizar uma avaliação mais sistemática desse modelo de ensino para averiguar as suas reais potencialidades e limitações.

\section{Referências bibliográficas}

ABED Citação de referências e documentos eletrônicos. Disponível em http://www2.abed.org.br/noticia.asp?Noticia_ID=275 Acesso em: 09 jun. 1997.

ADAO, Carlos e BERNADINO, Jorge. Blended-learning no Ensino de Engenharia: Um caso prático. Conferência CHALLENGES 2003, III Conferência Internacional sobre Tecnologias de Informação e Comunicação na Educação e $5^{\circ}$ SIIE, Simpósio Internacional em Informática Educativa, Braga 17, 18 e 19 de Setembro de 2003.

MORAN, J.M. Os novos espaços de atuação do professor com as tecnologias. IN:Anais do $12^{\circ}$ Endipe - Encontro Nacional de Didática e Prática de Ensino, in ROMANOWSKI, Joana Paulin et al (Orgs). Conhecimento local e conhecimento 
universal: Diversidade, mídias e tecnologias na educação. vol 2, Curitiba, Champagnat, 2004, páginas 245-253.

MOREIRA, M. A. Mapas Conceituais e Aprendizagem Significativa (Concept maps and meaningful learning). Adaptado e atualizado, em 1997, de um trabalho com o mesmo título publicado em O ENSINO, Revista Galáico Portuguesa de SócioPedagogia e Sócio-Lingüística, Pontevedra/Galícia/Espanha e Braga/Portugal, $\mathrm{N}^{\circ} 23$ a 28: 87-95, 1988. Publicado também em Cadernos do Aplicação, 11(2): 143-156, 1998. Revisado e publicado em espanhol, em 2005, na Revista Chilena de Educação Científica, 4(2): 38-44. Disponível em $<$ http://www.if.ufrgs.br/ moreira/mapasport.pdf>. Acesso em 12/03/2008.

NETO, A. C. C. e CASTANHO, M. J. P. Uso de um sistema baseado em regras fuzzy para avaliar a qualidade da cerâmica vermelha. In: Revista Eletrônica Laton Sensu, 2008. ISSN: 1980-6116.

PEGDEN, C. D,; SHANNON, R. E.; SADOWSKI, R. P. Introduction to simulation using SIMAN. McGraw-Hill, NY. 2 ed., 1990.

PELIZZARI, A.; KRIEGL, M.L.; BARON, M.P.; FINCK, N.T.L \& DOROCINSKI, S. I. Teoria da Aprendizagem Significativa Segundo Ausubel. Revista PEC, Curitiba.,v. 2, n. $1.37-42$ p. 2001/2002. 